\title{
Propagation of a Positive Streamer Toward a Dielectric Tip in Pure Nitrogen and in Air Under Voltage Pulses With Subnanosecond Rise Time
}

\author{
Anna Dubinova, Jannis Teunissen, and Ute Ebert
}

\begin{abstract}
We simulate a positive streamer discharge approaching a dielectric tip (e.g., of a dielectric rod) under a voltage pulse with a subnanosecond rise time. In air, the streamer reaches the tip, whereas in nitrogen, it does not.
\end{abstract}

Index Terms-Dielectrics, discharges (electric), plasma simulation.

$\mathbf{I}$ $\mathrm{N}$ OUR simulations, we use the convection-diffusionreaction model in local field approximation [1]. The ions are assumed immobile on the timescale of interest. The electrons can drift in an external electric field, diffuse, and ionize an ambient gas due to impact ionization (and due to photoionization in air). Once an electron reaches a dielectric surface, we assume that it stays there building up a surface charge and also no electron emission from the dielectric surface. The transport equations are coupled to the Poisson equation, which is solved in the whole simulation domain, including the electrode and dielectric. The ghost fluid method is implemented to discretize the Poisson equation near dielectric or conductive interfaces [2]. In the radial direction, we assume Neumann boundary conditions.

To study a positive streamer discharge interacting with a dielectric, we design an axially symmetric experiment in which a positive streamer propagates from a pin electrode down toward a dielectric tip (dielectric permittivity is 5) in a gas at $250 \mathrm{mbar}$ and $300 \mathrm{~K}$. The electrode (black areas in Figs. 1 and 2) and dielectric (white areas) are parametrized as spheroids, but any other shape could be chosen as well. The radii of the electrode and dielectric tip are 1.5 and $2 \mathrm{~mm}$, respectively, and their length is $4 \mathrm{~mm}$. The size of the simulation domain is $5 \mathrm{~mm} \times 12 \mathrm{~mm}$. The bottom of the domain is grounded and the ceiling with the pin protruding from it is under $10 \mathrm{kV}$, which is applied instantaneously. In Figs. 1 and 2, we zoom into the area of the streamer propagation. The streamer is launched by placing an electrically neutral Gaussian plasma seed of $0.5 \mathrm{~mm}$ radius

Manuscript received November 1, 2013; revised January 30, 2014 and August 22, 2014; accepted September 16, 2014. Date of current version October 21, 2014. The work was supported by the Technologiestichting STW Project under Grant 12119 through ABB Group, Eindhoven, The Netherlands.

The authors are with Centrum Wiskunde and Informatica, Amsterdam 1098 XG, The Netherlands (e-mail: a.dubinova@cwi.nl; jannis.teunissen@cwi.nl; ute.ebert@cwi.nl).

Color versions of one or more of the figures in this paper are available online at http://ieeexplore.ieee.org.

Digital Object Identifier 10.1109/TPS.2014.2360314 and $4 \cdot 10^{7} \mathrm{~cm}^{-3}$ maximal density at the tip of the electrode.

For their propagation, positive streamers, unlike negative streamers, need a source of free electrons ahead of the streamer. In nitrogen, this source could be, for example, leftover preionization in repetitive discharges. In our simulations, the value of the initial electron and ion density is $10^{4} \mathrm{~cm}^{-3}$, which corresponds to a discharge repetition frequency [3] of $\sim 0.3 \mathrm{~Hz}$. In air, the main source of free electrons is photoionization. It is an intrinsically nonlocal effect continuously providing free electrons ahead of the streamer. Photoionization is implemented according to Zheleznyak's model [4] using the computational approximations of [5].

In Figs. 1 and 2, we show the evolution of the electric field strength and the decimal logarithm of the electron density in an axial cross section. In pure nitrogen (Fig. 1), the electrons of the preionization drift upward in the external electric field depleting an area above the dielectric tip. The area is depleted independently of the actual preionization level. Positive streamers cannot penetrate into such areas. The streamer in Fig. 1, therefore, cannot reach the dielectric tip. A similar effect was observed in recent experiments [6]. There, it was shown that weak preionization, which does not influence the electric field, can guide positive streamers in nitrogen in directions perpendicular to the electric field lines.

In air (Fig. 2), UV photons released by excited nitrogen molecules can ionize oxygen molecules and continuously provide free electrons ahead of the streamer contributing to the background ionization. The streamer can now propagate all the way to the dielectric tip, touch it, and move around it.

In conclusion, we expect to observe electron-depleted regions that are avoided by positive discharges provided photoionization is negligible, surface electron emission during the voltage pulse is negligible, and voltage rises fast enough to neglect the source of free electrons in the depleted area (e.g., due to cosmic rays). With subnanosecond voltage being achievable, such conditions are more likely to be met in experiments.

\section{REFERENCES}

[1] U. Ebert et al., "The multiscale nature of streamers," Plasma Sour. Sci. Technol., vol. 15, no. 2, pp. S118-S129, 2006.

[2] S. Celestin, Z. Bonaventura, B. Zeghondy, A. Bourdon, and P. Ségur, "The use of the ghost fluid method for Poisson's equation to simulate streamer propagation in point-to-plane and point-to-point geometries," J. Phys. D, Appl. Phys., vol. 42, no. 6, p. 065203 , 2009. 

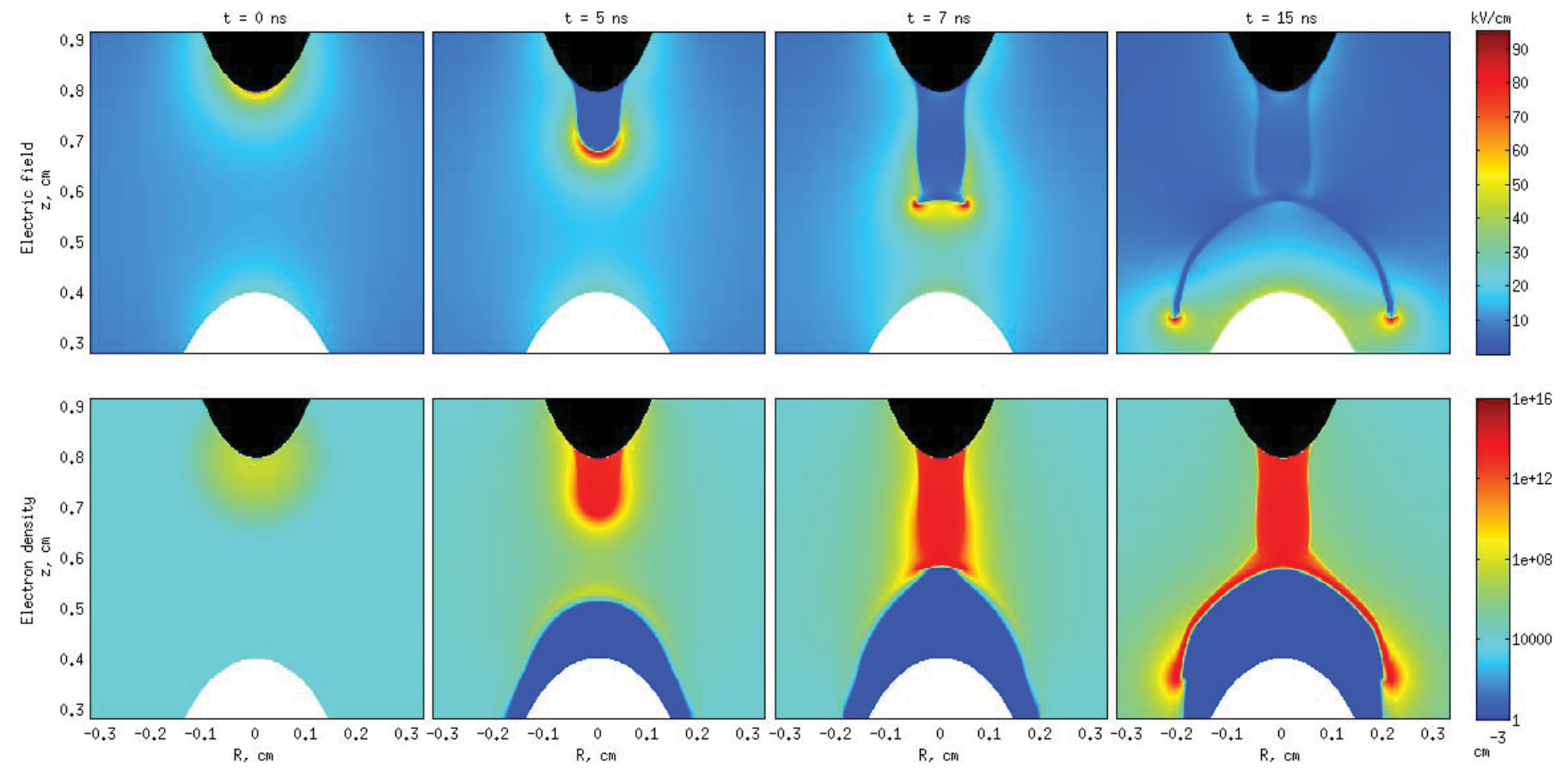

Fig. 1. Propagation of a positive streamer in pure nitrogen from an electrode (black areas) toward a dielectric tip (white areas). Evolution of the electric field strength (top panels) and logarithm of the electron density (bottom panels). The electrons of the preionization drift upward in the external electric field depleting the area above the dielectric tip (blue areas). Positive streamers in pure nitrogen avoid electron-free areas. The streamer does not reach the dielectric tip but surrounds the depleted area.
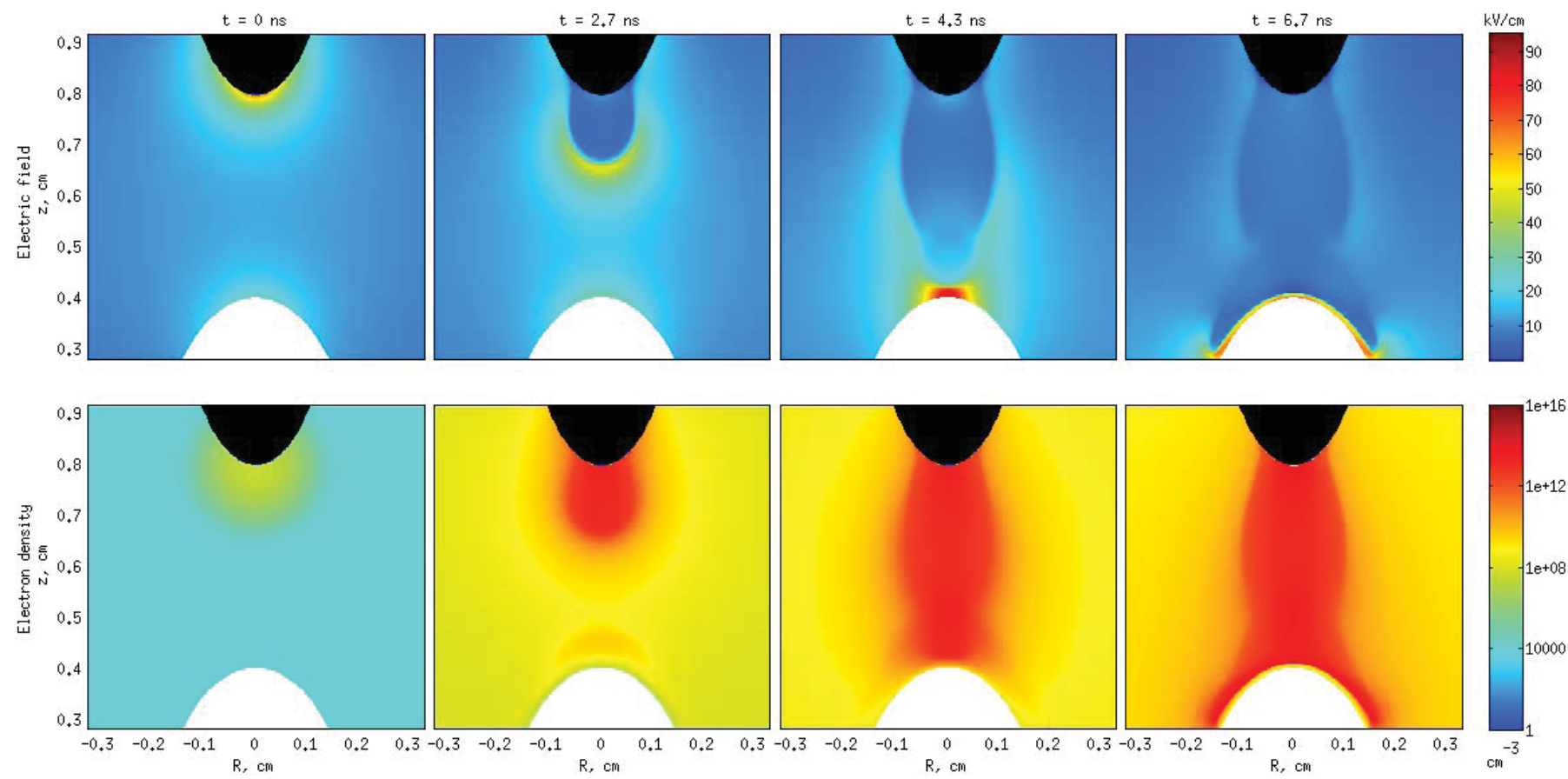

Fig. 2. Same situation, but now in air. Due to photoionization, the discharge continuously produces free electrons in front of the streamer head. Therefore, the streamer can propagate all the way to the dielectric tip, touch it, and move along it. The layer of high electric field around the tip of the dielectric after $6.7 \mathrm{~ns}$ forms when the space charge of the streamer head hits the top of the dielectric. In this layer, the electrons drift in the electric field leaving the positive ions with their space charge behind.

[3] S. Nijdam, G. Wormeester, E. M. van Veldhuizen, and U. Ebert, "Probing background ionization: Positive streamers with varying pulse repetition rate and with a radioactive admixture," J. Phys. D, Appl. Phys., vol. 44, no. 45, p. 455201, 2011.

[4] M. B. Zhelezniak, A. K. Mnatsakanian, and S. V. Sizykh, "Photoionization of nitrogen and oxygen mixtures by radiation from a gas discharge," High Temperature Sci., vol. 20, no. 3, pp. 357-362, 1982.
[5] A. Luque, U. Ebert, C. Montijn, and W. Hundsdorfer, "Photoionization in negative streamers: Fast computations and two propagation modes," Appl. Phys. Lett., vol. 90, no. 8, p. 081501, 2007.

[6] S. Nijdam, E. Takahashi, J. Teunissen, and U. Ebert, "Streamer discharges can move perpendicularly to the electric field," New J. Phys., to be published. 